\title{
Síndrome de Neuhäusser: Megalocórnea y retraso mental
}

\author{
Salesa Barja Y.'; Jorge Alvear A. '; Fanny Cortés M. ${ }^{2}$
}

\section{Neuhäusser syndrome}

A parent with megaloromea, de ayed osychomolor develofment, short stoture, congenital hypotonia, seizures and macrocephaly and mento retardation syndrome is described. These findings are compatiole with Neuhäusser's megalocornea and mentol relardation syndrome, rype 3 of Verloes. This rare entily was firsl reported by Neuhäusser in 1975 and shows an quiosomic recessive pallern oz interitonce even though sporadic cases have beer described.

(Key words: megalocomeo, mental retardation, Neuhäusser syndrome.)

El síndrome de megalocónea con retraso mental es una afección monogénica autosómica recesiva. Fue descrito por primera vez en 1975 por Neuhäusser ${ }^{1}$, que delineó las características fenotípicas de siete pacientes, tres de ellos hermanos, con megalocornea, retraso mental, anomalías neurológicas y retraso de crecimiento postnatal. Previamente en 1973, Frank ${ }^{2}$ había publicado un caso similar, en una niña beduina, hija de padres consanguíneos (primos de primer grado), con anomalías craneofaciales y esqueléticas. Desde entonces se han comunicado 24 casos en la literatura ${ }^{3-10}$. Hasta la fecha no se ha asignado el gen a un locus específico, si bien estudios de vinculación ("linkage") han permitido determinar que el gen de la megalocórnea ligado al cromosoma $X$ se encuentra en la región Xq13-q25 y con mayor probabilidad en $\mathrm{Xq} 21-\mathrm{q} 22^{11}$

La megalocórnea es un defecto del desarroHo ocular caracterizado por córneas de diámetro mayor que $12,5 \mathrm{~cm}$, simétricas y cuyo aumento no es progresivo ni afecta la presión intraocular $^{12}$. Puede ser aislado o asociado a otras anomalías del segmento anterior del ojo, como hipoplasia del iris o disgenesia iridocorneal. En esta última puede haber en la cốrnea una distro-

1. CONIN CREDES. Centro Pedro de Valdivia.

2. Unidad de Genética y Enfermedades Metabolicas. Instituto de Nutrición y Tecnologia de los Aljmentos (INTA), Universidad de Chile. fia típica en mosaico. En la mayoría de los casos de megalocórnea la herencia es ligada al cromosoma $X$, pero también han sido descritos algunos en que es autosómica recesiva o autosomica dominante ${ }^{13}$.

\section{Caso Clínico}

Niña de 9 meses, nacida del priner embarazo de su madre de 21 afos, quien es portadora de bocio eutiroideo. Padre sano de 22 años de edad. Sin antecedentes de consanguinidad ni enfermedades familiares de importancia. La madre sufrió de hipertensión arterial leve en el rercer trimestre del embarazo, no inginí alcohol, tabaco o drogas dnrante la gestación. La paciente nació por cesárea a las 38 semanas de gestacion. Peso al nacer $31,50 \mathrm{~g}$. su talla era $48 \mathrm{~cm}$. su perimetro craneano $36,5 \mathrm{~cm}$, Apgar 9 uno y cinco mínutos después de nacer. A las $9 \mathrm{~h}$ de vida mostró signos de bronconeumonía, la que respondio bien al tratamiento. Recibió alimentación natural por pocos días y fue dada de alta con fórmula láctea modificada. Al tercer mes de vida pesaba $4000 \mathrm{~g}$. medía $57 \mathrm{~cm}$ y se anotaba aumento de tamaño del ojo izquierdo. A los 7 y 8 meses de edad sufrió bronconeumonías, por las que fue necesario hospitalizarla. A la edad de nueve meses fue admitida a una unidad de recupecación nutricional con peso $4160 \mathrm{~g}$ (bajo el 3 percentil del Centro Estadounidense de Estadisticas de Salud, NCHS ), talla $62,5 \mathrm{~cm}$ (bajo p3 NCHS), circunferencia de cráneo $42.3 \mathrm{~cm}$ (bajo el p3 NCHS), peso/edad < $3 \mathrm{DE}$, tallaledad $<3$ DE. Poco activa, conectada al medio, macrocefalia relativa, frente abombada, cejas ralas, orejas de implantación baja, puente nasal deprimido, asimetría ocular (polo anterior de ojo izquierdo pmminente), ambas córneas aumentadas de diámetro $(12,5 \mathrm{~mm}$ cómea derecha y 13 $\mathrm{mm}$ la izquierda); presión intraocular ] l y $14 \mathrm{~mm} \mathrm{Hg}$ respectivamente (normal para la edad); fondo de ojos normal. Abdomen abombado, mancha "café con leche" paraum. 
bilical, hernia umbilical reductible. Sin defectos en tos genitales, columna y extremidades. Escaso panículo adiposo, pliegues cutáneos redundantes, poco desatrollo de los másculos esqueléticos, tono muscular muy disminuido, especialmente en las extremidades interiores; reflejos osteotendinoses no alterados. Dermatoglifos normales. Retraso desarrollo psicomotor severo con mayor compromiso de área molora gruesa según pruebas de Denver. Radiograías de cráneo, ultrasonografía y tomograf́a axial computadorizada del cerebro. anninoaciduria, aminoacidemia. rastreo metabólico. cromatografía de azúcares, creatina fosfoquinasa sérica, gases en sangre venosa, electrolitos plasmaticos, glicemia, hormonas tiroideas, ultrasonografias cardiaca $y$ abdominal normales. En el electroencefalograma se registraron ondas lentas y agudas ocasionales de localización anterior. En el hemograma había signos de anemia ferropriva leve que respondio al tratamiento. El estudio cromosómico con bandeo de alta resolución dio como resultado carjotipo $46, \mathrm{XX}$ sin anomalías.

Se alimentó exclusivamente por la boca, con dieta hiperproteica e hipercalórica. Su evolución nutricional fue favorable, con velocidad normal de crecimiento deJ perjmetro craneano, pero sin cambios de importancia en el desarrollo psicomotor, manteniéndose poco activa pero con buen conlacto social. Sufrió varios episodios de apnea simple, inmotivados, tno de los cuales se complicó con convulsiones tónicas y clónicas generalizadas, por lo que se inicio tratamieoto con fenobarbital.

\section{Comentario}

La megalocórnea es frecuentemente diagnosticada en oftalmología, generalmente asociada a glaucoma congénito. También forma parte de las características fenotípicas habituales de varios síndromes genéticos como el síndrome de Marfán, el stindrome de Weill-Marchesani, la osteogénesis imperfecta, el síndrome de ParryRomberg. la hiperglicinemia no quetótica. Se la encuentra con frecuencia mayor a la esperada por el azar, a craneoestenosis, diabetes mellitus, hipoacusia sensorioneural y trisomía 21 . Además se han descrito varios otros síndromes que incluyen glaucoma y megalocórnea secundaria entre sus manifestaciones.

En la tabla se señalan las manifestaciones fenolípicas más importantes de los pacientes con

Tabla

Caracleristicas descritas en 25 pacientes con síndrome de MMR

\begin{tabular}{|c|c|c|}
\hline Generales & $\begin{array}{l}\text { Edad (años) } \\
\text { Sexo femenino } \\
\text { Retraso mental } \\
\text { Talla baja }\end{array}$ & $\begin{array}{l}4,68 \pm 3.8 \mathrm{DE} \\
11 / 25^{*} \\
25 / 25^{*} \\
13 / 25^{*}\end{array}$ \\
\hline Oculares & $\begin{array}{l}\text { Megalocórnea } \\
\text { Hipoplasia del iris } \\
\text { Miopía }\end{array}$ & $\begin{array}{l}25 / 25^{*} \\
6 / 25 \\
4 / 25\end{array}$ \\
\hline Crane ofaciales & $\begin{array}{l}\text { Microccfalia } \\
\text { Macrocefalia } \\
\text { Facie redondeada } \\
\text { Frente abombada } \\
\text { Base nasal ancha } \\
\text { Hipertelorisıno } \\
\text { Epicanto } \\
\text { Globos oculares prominentes } \\
\text { Alteraciones auriculares } \\
\text { Labio superior largo } \\
\text { Micrognatia }\end{array}$ & $\begin{array}{l}5 / 25 \\
4 / 14^{*} \\
7 / 18^{*} \\
13 / 23^{*} \\
15 / 23^{*} \\
8 / 24^{*} \\
6 / 23 \\
6 / 21^{*} \\
9 / 21^{*} \\
9 / 21^{*} \\
10 / 20^{*}\end{array}$ \\
\hline Neurológicas & $\begin{array}{l}\text { Retraso motor } \\
\text { Hipotonia congenita } \\
\text { Convulsiones } \\
\text { Ataxia } \\
\text { Movimientos anormales }\end{array}$ & $\begin{array}{l}24 / 25^{*} \\
21 / 22^{*} \\
7 / 22^{*} \\
4 / 22 \\
4 / 22\end{array}$ \\
\hline Esqueléticas & $\begin{array}{l}\text { Alteraciones digitales } \\
\text { Xifoescoliosis } \\
\text { Genu varo } \\
\text { Pie plano valgo }\end{array}$ & $\begin{array}{l}5 / 8 \\
8 / 24 \\
5 / 21 \\
7 / 21\end{array}$ \\
\hline
\end{tabular}

* Alteraciones presentes en la paciente de este informe. 


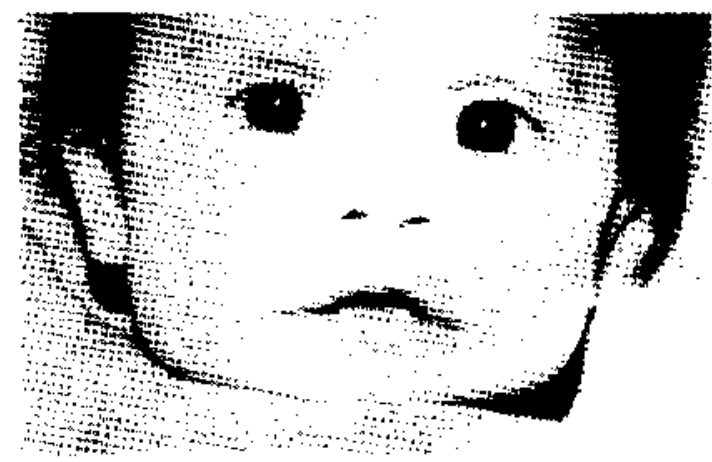

Figura 1: Aspecto facial de una nifia con sindrome de Neuhäusser: megalocórnea, frente prominente, puente nasal ancho y bajo, implantación baja de las orejas, labio superior largo y micrognatia.

esta afección publicados hasta la fecha, incluyendo nuestra paciente. En las primeras comunicaciones se consideraban, como criterios mínimos para el diagnóstico del síndrome. la existencia de megalocórnea, retraso mental y retraso de crecimiento, sin embargo, las alteraciones neurológicas, especialmente la hipotonía, se registran en la mayoría de los pacientes publicados.

En 1993 Verioes y col. ${ }^{6}$ propusieron una clasificación del síndrome de megalocórnea con retardo mental en cuatro tipos, de acuerdo a las características fenotípicas de los pacientes reconocidos hasta entonces: el tipo 1 (síndrome de Neuhäusser) corresponde a pacientes con megalocórnea, hipoplasia del iris, retraso mental, dismorfias craneofaciales menores y sindrome convulsivo. El tipo 2 (Frank-Temtamy) es el de pacientes con megalocórnea sin otras alteraciones de la cámara anterior, retraso mental, camptodactilia, escoliosis y retraso de crecimiento. Los pacientes del tipo 3 (Verloes) tienen megalocórnea, iris normal, macrocefalia relativa o absoluta, retraso mental (con especial compromiso del desarrollo motor), hipotonía, convulsiones y dismorfias (abombamiento frontal, nariz prominente de base ancha, fisuras palpebrales antimongoloides, hipoplasia malar, retrognatismo, tórax angosto, dedos finos y largos). En el tipo 4 (Frydman) los afectados tienen megalocórnea, aumento del perímetro craneano, retraso mental, dismorfias similares a las descritas en el tipo 3, mas anomalías de los pabellones auriculares y obesidad.
Los tipos 1,2 y 4 muestran claramente un patrón hereditario autosómico recesivo: en cambio los pacientes con fenotipo 3 parecen ser de ocurrencia espotádica y no tener un riesgo aumentado de recurrencia. A pesar de la gran dispersión de las características clínicas de los pacientes con el síndrome de megalocórnea y retardo mental, la clasificación preliminar mencionada es de utilidad para aproximarse al diagnóstico ante pacientes con una afección clínicamente tar heterogénea. La paciente que se comenta correspondería al tipo 3 a de Verloes, de ocurrencia esporadica, sin aumento del riesgo de recurrencias en futuros embarazos. Su pronóstico, dada la baja ocutrencia de la afección, no es precisable.

Indudablemente, es necesario avanzar en el estudio etiológico definitivo de todo paciente con megalocórnea con el fin de ofrecer una orientación pronóstica a los pacientes y sus familias. Lo anterior sólo será posible en la medida que se busquen, diagnostiquen y publiquen casos adicionales que permitan delinear adecuadamente la afección. que es el motivo principal de la presente publicación.

\section{Resumen}

Se describe una niña de 12 meses de edad, con megalocórnea, retraso severo del desartollo psicomotor, desnutrición calorico proteica de tercer grado: sîndrome hipotónico, retraso de crecimiento, macrocefalia relativa y convulsiones, síntomas y signos que corresponden a los del sindrome de Neuhäusser tipo 3 de Verloes. Este síndrome tiene como características más importantes megalocórnea, retraso mental, trastornos neurológicos y retraso de crecimiento, las que con frecuencia se asocian con otras anomalías menores, $y$ en lo que respecta a la herencia, ésta es preferentemente autosómica recesiva, pero puede haber casos esporádicos.

(Palabras clave: megalocornea, retardo mental, síndrome de Neuhäusser.)

\section{Referencias}

1. Neuhausser G. Kaveggia EG. France TD. Opiz JM: Syndrome of mental retardation, seizures, hypotonic cerebtal palsy, and megalocomea, recessively inherited. $Z$ Kinderheilk 1975; 120: 1-18. 
2. Frank Y. Ziprkowiski $M$, Romano A, et al: Megalocornea associated with multiple skelekal anomalies: A new genetic syndrome? J Genet Hum 1973: 21: 67.72.

3. Schmidt $\boldsymbol{R}$, Rabin $I$ : The syndrome of mental retardation and megalocomea (Absiract) Am J Human Genet 1981: 33: 90A.

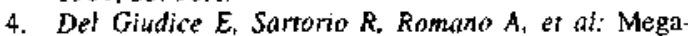
locornea and mental retardation syndrome: Two new cases. Am J Med Genet 1989; 26: 417-420.

5. Gronberg-Jensen $M$ : Megaloconea and mental re tardation syndrome: A new case. Am J Med Genet $1989 ; 32: 468-469$.

6. Verloes A, Jowind H, Elmer C. et al: Heterogeneity versus variability in megalocornea mental retardation syndromes: Report of new cases and delineation of 4 probable Types: Am J Med Genet 1993; 46:132-137.

7. Ratas-Rosthschild A. Berkenstadt M, Goodmann RM: Megalocornea and mental retardation syndrome. Am J Med Genet 1988; 29: 221-222.

8. Santotaya JM, Grijalbo A, Delgado A. Endozain G: Aditional case of Neuhäusser megalocomea and men- tal retardation syndrome. Am J Med Genet 1992; 43: 609-611.

9. Frydman M, Berkenstad M, Raas-Rothchild A. Goodman $R M$ : Megalocornea, macrocephaly, mental and motor retardation. Clin Genet 1990: 38: 149-1\$4.

10. Temiamy S, Abdel-Hamid J, Hussein F, el al: Megalocomea, mental retardation syndrome (MMR). Delineation of a new entity (MMR 2). Am J Human Genet $1991 ; 49: 125 \mathrm{~A}$.

11. Mere FM. Bleeker-Wagemakers EM. Ochler M, Gal A. Delleman JW: X-linked megalocornea. Ocular findings and linkage analysis. Ophthalmic Paediatr Genet $1991 ; 12: 153-157$.

12. Sugar J: Defects of the comea. In Emery AEH, Rimoin DL (eds): Principles and Practice of Medical Genetics: Churchill Livingstone Edinburgh 1983: 497508.

I3. MCKusick V: Mendelian Inheritance in Man. Catalogs of autosomal dominant, autosomal recessive and $\mathrm{X}$ linked phenotypes. Ninth Edition. The Johns Hopkins Unjversity Press, Baltimore and London, 1992

\section{AVISO A LOS AUTORES}

Con el objeto de dar prioridad a los trabajos de investigación, en vista de las limitaciones de espacio de la Revista Chilena de Pediatría, el Comité Editorial ha acordado restringir la impresión de casos clínicos a un máximo de dos por cada número. 